\title{
RHEUMATOLOGY
}

\section{Management of childhood arthritis. Part 1: acute arthritis}

\author{
P N Malleson
}

This is the first article in a series on rheumatology.

\footnotetext{
Department of Paediatrics, The Research Centre, Vancouver, Canada

Correspondence to: Dr P N Malleson, Paediatric Rheumatology, Faculty of Medicine, Department of Paediatrics, The Research Centre, 950 West 28th Avenue, Vancouver, British Columbia, Canada V5Z $4 \mathrm{HA}$.
}

Arthritis in childhood is not uncommon. Estimates of the annual incidence of arthritis vary widely, but for all forms of chronic arthritis it is probably between 5 and 10 per 100000 children aged 16 years or younger. ${ }^{12}$ Acute forms of arthritis are perhaps four times more common. ${ }^{3}$ Most paediatricians will therefore see several patients with arthritis each year. The aim of this paper and part 2 of the series ${ }^{4}$ is to give a concise, but moderately comprehensive, overview of this topic which will help in the management of this heterogeneous group of disorders.

Although chronic arthritis can present with a sudden and painful onset of one or more swollen joints, such a presentation should raise suspicions that the arthritis is due to trauma or to a joint or contiguous bone infection.

\section{Trauma}

A traumatic cause of joint swelling is usually obvious from the patient's history, but parents sometimes incorrectly presume that a swollen joint must have been due to an injury. It is unlikely that an injury is the cause of a swollen joint if the child has not had a definite traumatic event that was painful enough to immediately prevent the child continuing with the activity. The exception to this is perhaps a child with haemophilia, who might develop a haemarthrosis after only mild trauma.

If it is uncertain whether the swelling of a joint is due to a joint effusion or simply due to soft tissue swelling, an ultrasound examination of the joint can be helpful and may also be able to determine whether there is blood in the joint. Synovial fluid analysis of a traumatic effusion usually shows a low white cell count $\left(<0.2 \times 10^{9} / 1\right)$, but this can occasionally be much higher and mimic fluid from a septic joint. A haemarthrosis raises the possibility of either a fracture into the joint or a torn anterior cruciate ligament. The presence of marrow fat is indicative of a fracture.

Magnetic resonance imaging has revolutionised the investigation of musculoskeletal disorders and it has largely replaced arthrograms in the investigation of joints affected by trauma. Magnetic resonance imaging should only be performed after a plain radiograph has been taken (it is embarrassing to diagnose a fracture on magnetic resonance imaging that would have been perfectly visible on a much cheaper radiograph!).

\section{Septic arthritis}

Septic arthritis is, of course, a medical emergency and if suspected must be investigated with both blood cultures and arthrocentesis for Gram stain, cultures, and tests for specific infectious antigens. Although a high peripheral white cell count, high erythrocyte sedimentation rate, and high synovial fluid white cell count $\left(>5.0 \times 10^{9} / 1\right)$ is suggestive of an infection, similar findings can occur in reactive arthropathies and even in early chronic arthropathies. Furthermore, synovial white cell counts may be only mildly increased in proved septic joints. A significant proportion of children with probable septic arthritis have persistently negative cultures (even without preceding antibiotic treatment) and negative antigen tests. ${ }^{5}$ Therefore it is probably necessary to treat a few children who may not actually have septic arthritis with intravenous antibiotics so as not to miss a child who does have this disorder. Although septic arthritis usually affects only a single joint (usually a knee) in children, $4-6 \%$ of patients will have two or more joints affected. Therefore the fact that several joints are swollen should not lead to the exclusion of the possibility of sepsis.

It is fairly common, particularly in neonates, for an osteomyelitis to have an associated arthritis, which may or may not be septic. Bone tenderness beyond the margins of the joint is suggestive of osteomyelitis. Radiographs often show soft tissue changes adjacent to an infected bone several days before bone changes become visible. A bone scan is usually diagnostic of osteomyelitis even if the joint has already been aspirated.

The initial treatment of septic arthritis should be with an antibiotic given intravenously, such as cefuroxime, which is effective against Staphylococcus aureus, streptococci, and, if the child has not been immunised, Haemophilus influenzae type $\mathrm{b}$, with the antibiotics being changed as appropriate once the organism has been cultured and its sensitivities determined. In a child with septic arthritis of the foot, consideration should be given to using an antibiotic effective against Pseudomonas aeruginosa. The total duration of treatment should be $4-6$ weeks, guided by the clinical 
signs, peripheral white cell count, and erythrocyte sedimentation rate. The use of antibiotics given by mouth in high doses once the infection is under control has been advocated, but requires careful monitoring of compliance (ideally with serum bactericidal titres) and clinical progress. ${ }^{67}$ Although most authorities advocate open drainage of a septic hip joint and repeated aspirations of other joints, there is little evidence supporting repeat aspirations. ${ }^{8}$ They are probably unnecessary if the child's course progresses satisfactorily.

It is extremely important to provide adequate pain relief (something that is often forgotten); this should usually initially be with intravenous morphine. The use of a splint to 'rest' and protect the joint is appropriate for a few days, but should not be prolonged as it will encourage joint contractures and muscle wasting. The role of non-steroidal antiinflammatory drugs (NSAIDs) has not been formally evaluated, but is probably helpful. Whether the use of corticosteroids early in the disease course might be of benefit, as has been shown for bacterial meningitis, ${ }^{9}$ is unknown. Occasionally the inflammatory response appears to persist long after it seems clear that the infection has been eradicated, and then the use of NSAIDs or intra-articular corticosteroids, or both, seems justified, as it is probably the inflammatory response rather than the bacteria themselves that is damaging to the joint cartilage.

\section{Lyme arthritis}

Lyme arthritis is a form of infectious arthritis which is common in some areas of the world. It occurs several weeks to months after the initial infection with Borrelia burgdorferi. It has an acute oligoarticular onset and tends to be episodic, with each episode lasting a few days. The episodic nature of the arthritis is an important clue that this is not idiopathic chronic arthritis. Treatment with erythromycin $30 \mathrm{mg} / \mathrm{kg} /$ day or amoxicillin $30-50 \mathrm{mg} / \mathrm{kg} /$ day by mouth, both divided twice a day, is usually effective. If untreated the arthritis can recur several times a year for several years, but the episodes gradually decrease in frequency and eventually resolve completely. ${ }^{10}$

A few children, whether they have been treated appropriately or not, go on to develop chronic arthritis. This outcome is associated with the presence of antibodies to HLA-DR4 or HLA-DR2 and with high antibody titres to the outer surface protein A of Borrelia burgdorferi. ${ }^{11}$ The problem with managing possible Lyme arthritis is that the diagnosis can be difficult. Only about $40-70 \%$ of children have the characteristic erythema migrans rash, constitutional symptoms may be mild, and serological tests are difficult to interpret as there is a high incidence of false positivity. Paediatricians should be wary of treating children with arthritis without a history of tick bites or erythema migrans for Lyme disease, unless there is western blot confirmation of a positive serology, as the risk of antibiotic toxicity is probably higher than the risk of complications from the untreated disease. ${ }^{12}$ It is worth noting that in areas where the disease is endemic, many children diagnosed as having chronic Lyme disease actually have fibromyalgia. ${ }^{13}$

\section{Reactive and postinfectious arthritis}

An acute arthritis after an infection in which there is no evidence of a septic process of the joint is considerably more common than septic arthritis. ${ }^{3}$ The classical postinfectious arthropathy is rheumatic fever. In recent years a form of poststreptococcal arthritis has been recognised that is more polyarticular and more prolonged than in typical acute rheumatic fever and which does not always fulfil the criteria for rheumatic fever, but which may be complicated by carditis. ${ }^{14}$ It should be suspected in any child who has a sore throat or constitutional symptoms at the onset of the arthritis and should be investigated with throat cultures and antibodies to streptococcus. If a recent streptococcal infection can be proved, then cardiac evaluation with echocardiography should be undertaken. Penicillin prophylaxis is mandatory in children with evidence of carditis, but is controversial if there is no clinical or echocardiographic evidence that the heart is affected.

Arthritis secondary to various enteric infections is not rare in children. A family history of spondyloarthropathy is often present and most patients are positive for HLA-B27. The arthritis can be extremely painful, mimicking sepsis; it is usually relatively short lived, but may occasionally become chronic. Although there is some evidence that there may be persisting antigens in the joint, there is no clear evidence that antimicrobial treatment modifies the disease course. Treatment is with an NSAID such as tolmetin until the joint inflammation has resolved clinically and the complete blood count and erythrocyte sedimentation rate have normalised.

Chlamydia trachomatis infection of the genitourinary tract should be considered in any sexually active adolescent with new onset arthritis. Viable chlamydia may persist in the joints of patients with Reiter's syndrome ${ }^{15}$ and antibiotic treatment of the initial infection as well of the subsequent arthritis can be beneficial. ${ }^{16}$ Therefore antibiotics as well as NSAIDs are justified if this diagnosis is proved.

Many acute transient arthritides are probably due to viral infections. Rubella infection of both the wild type and after immunisation is a common cause of arthralgias or arthritis in adolescents, but is a rare cause in young children. Parvovirus B19 is also a common cause of acute arthritis in adolescents and young adults, but, like rubella, it rarely causes arthritis in young children. Arthritis can occur after chickenpox, but sometimes this can actually be due to a septic arthritis, presumably due to haematogenous spread from an infected lesion. As viral arthritides are self limiting the treatment is usually no more than a few days of NSAID treatment, combined with rest and other symptomatic measures.

\section{Malignancy}

After trauma and infection the most important cause of acute or recent onset arthritis is 
malignancy, not because it is a particularly common cause of arthritis, but because a delay in diagnosis may have such profound consequences both for the patient and for the doctor. Leukaemia and neuroblastoma can both present with true joint swelling. Bone tumours (and uncommonly tumours arising from joint tissues) can present with joint pain. The true cause of the arthropathy should be suspected if the child is constitutionally unwell, if there is bone tenderness rather than tenderness limited to the joint line, if there is profound anaemia or a cytopenia, or if the platelet count is not increased in the face of an increased erythrocyte sedimentation rate. High lactate dehydrogenase levels are a helpful clue that the child has leukaemia. ${ }^{17}$ The management of the arthritis associated with a malignancy is the management of the underlying disease, but the pain may well be severe enough to warrant intravenous morphine during the first few days of specific oncology treatment.

\section{'Rheumatology tests'}

Although doctors commonly order tests for rheumatoid factor and antinuclear antibodies in children with acute arthritis in an attempt to make a positive diagnosis of a rheumatic disease, this policy is incorrect and should be abandoned. When performed as a screening test rheumatoid factor tests are rarely positive, and when positive are as likely to be in children with other diseases as in children with chronic arthritis. ${ }^{18}$ Positive tests for antinuclear antibodies using HEp- 2 cells commonly occur, but are found as often in children with nonrheumatic disease as in those with rheumatic disease ( $\mathrm{P}$ Malleson et al, unpublished data). Positive tests for specific antinuclear antibodies can also occur in various malignancies and could lead to a delay in the correct diagnosis. ${ }^{19}$

\section{Conclusions}

Acute joint swelling is a disorder that can often be due to a serious underlying disease. It should not be dismissed as due to trauma or the first manifestations of a chronic arthritis without careful consideration of the relevant history and a judicious use of investigations. Although undoubtedly not required in every case, there should be a low threshold for performing an arthrocentesis. Doctors who would not hesitate to perform a lumbar puncture if they had even a mild suspicion that the child had meningitis often seem reluctant to perform arthrocentesis because of its painful nature when they suspect septic arthritis. With the use of 'conscious sedation' with midazolam and fentanyl, EMLA cream, and buffered lignocaine, an arthrocentesis should be almost free from pain and anxiety for the child, parent, and doctor.

I gratefully acknowledge the advice of Dr Richard Beauchamp and Dr Simon Dobson in the preparation of this paper.

1 Gäre BA, Fasth A. Epidemiology of juvenile chronic arthriis in southwestern Sweden: a 5-year prospective population study. F Pediatr 1992;90:950-8.

2 Malleson PN, Fung MY, Rosenberg AM, for the Canadian Pediatric Rheumatology Association. The incidence of pediatric rheumatic diseases: results from the Canadian Pediatric Rheumatology Association Disease Registry. 7 Rheumatol 1996;23:1981-7.

3 Kunnamo I, Kallio P, Pelkonen P. Incidence of arthritis in urban Finnish children. Arthritis Rheum 1986;29:1232-8.

4 Malleson P N. Management of childhood arthritis. Part 2: chronic arthritis. Arch Dis Child 1997 (in press).

5 Fink CW, Nelson JD. Septic arthritis and osteomyelitis in Fink CW, Nelson JD. Septic arthritis and osteomyelitis
children. Clinics in Rheumatic Diseases 1986;12:423-35.

6 Nelson JD, Bucholz RW, Kusmiesz H, Shelton S. Benefits and risks of sequential parenteral-oral cephalosporin therapy for suppurative bone and joint infections. $\mathcal{F}$ Pediatr Orthop 1982;2:255-62.

7 Dagan R. Management of acute hematogenous osteomyelitis and septic arthritis in the pediatric patient. Pediatr Infect Dis f 1993;12:88-92.

8 Nord KD, Dore DD, Deeney VF, et al. Evaluation of treatment modalities for septic arthritis with histological grading and analysis of levels of uronic acid, neutral protease, and interleukin-1. F Bone foint Surg Am 1995;77: $258-65$.

9 Odio CM, Faingezicht I, Paris M, et al. The beneficial effects of early dexamethasone administration in infants and children with bacterial meningitis. N Engl f Med 1991; 324:1525-31.

10 Szer IS, Taylor E, Steere AC. The long-term course of Lyme arthritis in children. N Engl f Med 1991;325:159-63.

11 Steere AC, Levin RE, Molloy PJ, et al. Treatment of Lyme arthritis. Arthritis Rheum 1994;37:878-88.

12 Magid D, Schwartz B, Craft J, Schwartz JS. Prevention of Lyme disease after tick bites. A cost-effectiveness analysis. N Engl f Med 1992;327:534-41.

13 Sigal LH, Patella SJ. Lyme arthritis as the incorrect diagnosis in pediatric and adolescent fibromyalgia. Pediatrics 1992;90:523-8.

14 Fink CW. The role of the streptococcus in poststreptococcal reactive arthritis and childhood polyarteritis nodosa. F Rheumatol 1991;18(suppl 29):14-20.

15 Rahman MU, Cheema MA, Schumacher HR, Hudson AP. Molecular evidence for the presence of chlamydia in the synovium of patients with Reiter's syndrome. Arthritis synovium of patients
Rheum 1992;35:521-9.

16 Lauhio A, Leirisalo-Repo M, Lähdevirta J, Saikku P, Repo H. Double-blind, placebo-controlled study of three-month reatment with lymecycline in reactive arthritis, with special reference to chlamydia arthritis. Arthritis Rheum 1991;34:614.

17 Wallendal M, Stork L, Hollister JR. The discriminating value of serum lactate dehydrogenase levels in children with malignant neoplasms presenting as joint pain. Arch Pediatr Adolesc Med 1996;150:70-3.

18 Eichenfield AH, Athreya BH, Doughty RA, Cebul RD. Utility of rheumatoid factor in the diagnosis of juvenile rheumatoid arthritis. Pediatrics 1986;78:480-4

19 Swissa M, Amital-Teplizki H, Haim N, Cohen Y, Shoenfeld Y. Autoantibodies in neoplasia. An unresolved enigma. Cancer 1990;65:2554-8. 